\title{
Effects of peripheral circular contours on dynamic spatial orientation
}

\author{
TIMOTHY G. BABLER \\ University of Wisconsin, Madison, Wisconsin \\ and \\ SHELDON M. EBENHOLTZ \\ State University of New York, New York, New York
}

\begin{abstract}
The rod-and-frame effect (RFE) was investigated with the use of a frame that oscillated about an axis at its center at five different frequencies, ranging from .013 to $.213 \mathrm{~Hz}$. The resultant RFE shifted continuously with the roll motion of the frame, and it was significantly larger at the lowest frequency $(.013 \mathrm{~Hz})$ than under comparable static conditions. The dynamic RFE was lowest at the higher oscillation frequencies. Oscillatory roll vection-apparent self-motion-was reported by 3 of the 9 subjects when the frame was oscillating at its highest frequency $(.213 \mathrm{~Hz})$. The subjects yielded large increases in the RFE during the sessions with reports of vection. Surrounding the kinetic frame with a circular contour eliminated all reports of vection and significantly interacted with frequency to reduce the RFE-but only at low frequencies. The reduction amounted to $21.2 \%$ averaged over all 9 subjects at the three lowest frequencies. A surrounding contour, therefore, suppressed low-frequency kinetic visual orientation information that might otherwise have produced larger changes in apparent self-orientation and perceived vertical. Vection-sensitive subjects differed from nonvection subjects by exhibiting (1) a high-frequency fall-off in real-motion gain, (2) a high-frequency enhancement in illusory-motion gain, and (3) only a small and nonsignificant increase in illusory-movement phase lag with increases in frequency.
\end{abstract}

An upright line enclosed within a tilted square frame will appear to be tilted in the direction opposite that of the frame. When attempting to set the line to an apparent vertical orientation, an upright observer will tend to err in the direction of the frame tilt. This illusory shift in verticality, known as the rod-and-frame effect (RFE), has been studied extensively since it was first described by Witkin and Asch (1948).

Recent evidence lends support to the interpretation of the RFE as an automatic noncognitive phenomenon, a type of visual-vestibular interaction that aids in self-orientation (Ebenholtz, 1977, 1985a; Ebenholtz \& Callan, 1980). In other words, the RFE is essentially an example of the work of the ambient visual system. According to the concept of two visual systems (Leibowitz \& Post, 1982), the focal system responds primarily to stimulation of the central visual fields, entails cognitive processing, and is responsible for the discrimination and identification of visual features. The ambient system, on the other hand, responds both to peripheral and central stimulation, in-

This research was presented by Timothy G. Babler in partial fulfillment of the requirements for the MS degree in the Department of Psychology, University of Wisconsin-Madison. The research was supported in part by Research Grant BNS-8201441 from the National Science Foundation, to Sheldon M. Ebenholtz. The authors thank Robert Post for his illuminating comments on a draft of the manuscript. Reprint requests should be directed to Sheldon M. Ebenholtz, Schnurmacher Institute for Vision Research, College of Optometry, State University of New York, 100 East 24th Street, New York, NY 10010. volves lower-order automatic processes, and is capable of regulating the perception of self-motion and body orientation.

The RFE does, indeed, respond to stimulation in the visual periphery. An increase in the eccentricity of the sides of the tilted frame produces an increase in the RFE (Ebenholtz, 1977; Ebenholtz \& Callan, 1980). In fact, changes in the visual angle of the display yield corresponding changes in the magnitude of the RFE. Since a frame producing a large retinal angle necessarily covers a greater retinal area in the periphery than does a smaller less eccentric image, the increase in the RFE may seem to be attributable to increased stimulus size or area, rather than to eccentricity as such. There is, in fact, evidence to support the role of image area or contour length, since a partial frame consisting either of corners only or of sides only (Ebenholtz, 1985a; Streibel, Barnes, Julness, \& Ebenholtz, 1980) produces a significantly lower RFE than the comparable full-frame configuration does. On the other hand, although corners represented only about $23 \%$ of the linear extent of the sides in these experiments, there were no significant differences between the two partialframe configurations. This might have occurred because the corners of a square frame are necessarily more eccentric than the sides, and hence the corners may have had the advantage of eccentricity to balance the loss due to area. Thus both eccentricity and contour length or area may be significant parameters of the RFE. It also is important to note that the RFE is sheltered from many of 
the cognitive processes associated with the focal system. Perceived size (Ebenholtz, 1977; Ebenholtz \& Callan, 1980), form (Streibel et al., 1980), and depth perception (Ebenholtz \& Glaser, 1982) do not influence the magnitude of the RFE.

The image of the tilted frame can be assumed to stimulate receptive fields in the visual periphery, which act later upon detectors that signal self-orientation. The end result of viewing the tilted frame is an illusory self-tilt in the direction opposite to the frame tilt. If the observer does feel tilted and the rod is aligned with the body, then the rod too should appear tilted away from vertical. When asked to adjust the rod to apparent vertical, the observer should then place the rod in the direction opposite to perceived self-tilt, which is toward the frame tilt.

A number of studies have demonstrated sensations of self-tilt that result from viewing a tilted frame. Ebenholtz and Benzschawel (1977) found errors of a few degrees in the direction of a tilted frame when subjects attempted to set their head to gravitational vertical. Later studies, using a similar procedure (Sigman, Goodenough, \& Flannagan, 1979) and a magnitude-estimation procedure (Sigman, Goodenough, \& Flannagan, 1978) have yielded similar self-tilt results. Since apparent self-tilt, however, has been shown to be systematically lower in magnitude than the corresponding RFE is, it would appear that the tilted frame represents a stimulus for both effects, but that induced self-tilt is not the singular cause of the RFE.

In recent years, a rather compelling means of dramatically altering the size of the RFE has been found. Merely surrounding the tilted frame with a circular contour reduces the tilt effect to only $25 \%$ of its original magnitude (Ebenholtz \& Utrie, 1982, 1983). If, instead, a necessarily smaller circle is inscribed within the frame, no RFE reduction occurs. However, with the outer circle and frame removed, a frame within the latter circle again will be inhibited from yielding a full RFE. Here the peripheral nature of the visual stimulus has become strictly relative, since the relative locations of the circle and frame, rather than eccentricity as such, have been shown to be crucial for the control of spatial orientation. If there is a separation in depth between the frame and circle, the inhibition of the RFE remains intact (Ebenholtz, 1985b). The cause of this inhibition effect has yet to be explained.

In the study described here, we examined the RFE under dynamic conditions. This was achieved by oscillating the frame at various frequencies. A kinetic rod-andframe device allows for a comparison between static and dynamic tilt effects. If the RFE is, indeed, an example of a static analog of visually induced self-motion (Ebenholtz, 1977), then a kinetic frame may be capable of inducing vection. In the case of a sinusoidally moving frame, oscillatory roll vection may result, similar to that induced by other rotating visual stimuli (see, e.g., Dichgans, Mauritz, Allum, \& Brandt, 1976). A second goal was to examine the inhibition effect of the surrounding circular contour under dynamic conditions.

\section{METHOD}

\section{Subjects}

The subjects were 6 female and 3 male students from the University of Wisconsin-Madison. Their ages ranged from 18 to 22 . Three additional subjects were eliminated, 1 because of motion-sickness symptoms (headache and nausea), and 2 others for failing to follow instructions. Since eyeglasses constitute a possible frame of reference for vertical orientation, the subjects were limited to people wearing contact lenses or with normal uncorrected vision.

\section{Apparatus}

Both the rod and the frame were made of electroluminescent panels. The square frame was $59.5 \mathrm{~cm}$ on each side, with a width of $3.8 \mathrm{~cm}$. Square pieces of black tape were placed on the frame panels to create a checkerboard-like pattern. The rod was $7.5 \times$ $0.3 \mathrm{~cm}$. The rod and frame were positioned within a luminous circle, whose inner diameter was $98.5 \mathrm{~cm}$ and width was $1.3 \mathrm{~cm}$. The frame, rod, and circle were all set to the same subjective luminance of about $.10 \mathrm{~cd} / \mathrm{m}^{2}$ and shared the same axis around the line of sight. The subjects viewed the display in the frontal plane from a distance of about $50.76 \mathrm{~cm}$, which provided, for the length of the rod and one side of the frame, retinal angles of $8.45^{\circ}$ and $60.75^{\circ}$, respectively, and for the diameter of the inner edge of the circle, a retinal angle of $88.27^{\circ}$.

The rod and frame could be sinusoidally oscillated about their centers at various frequencies and amplitudes. The frequencies ranged from 0 (static) to $.213 \mathrm{~Hz}$, while the peak to peak amplitudes ranged from $-15^{\circ}(\mathrm{ccw})$ to $+15^{\circ}(\mathrm{cw})$, symmetrically with respect to vertical. The motion of the rod could be controlled by the subject with a $30-\mathrm{cm}$ diameter steering wheel, calibrated with a gain of 1.0 , such that a $1^{\circ}$ wheel rotation produced a $1^{\circ}$ rod rotation.

The subjects sat in a modified dental chair. The chair's footrest was covered with foam rubber to minimize somatosensory cues. Attached to the chair was a helmet connected to a strain gauge, which was used to monitor any tilt of the head.

The position of the steering wheel, the frame position, the line orientation, and the subject's head position ${ }^{1}$ were all recorded on both a pen recorder and an analog signal recorder.

\section{Design}

Each subject was exposed to all 12 conditions. These included the five frame oscillation frequencies of $.013, .027, .053, .107$, and $.213 \mathrm{~Hz}$, plus a static frame, all with the circle illuminated, and the same 6 conditions with the circle absent. The sequence of conditions was determined by a balanced six-item Latin square. Within subjects, the circle and no-circle conditions were completed in successive sessions counterbalanced across subjects, so that each subject completed both circle and no-circle conditions at a given frequency before a different frequency was presented. The sessions were approximately 1 to 2 days apart, and only one frequency was examined per session.

\section{Procedure}

The subjects viewed the rod-and-frame display in complete darkness, so that only the line, frame, and circle were visible. With the subject seated, the steering-wheel box was placed across the arms of the chair in a horizontal plane. A black cloth was then draped across the subject's shoulders, arms, and hands, as well as across the steering-wheel box. This precaution was taken to minimize any possible visual orientation cues that the hands or box might have provided.

The subjects were instructed to keep the line looking vertical at all times. Vertical was defined for the subjects as parallel to the 
unseen walls and in the direction of gravity. Such a task required the subjects to make prompt and continuous steering adjustments throughout the entire session. In addition, the subjects were instructed to report immediately any detection of the frame's ceasing to oscillate or any sensation of self-motion. These were prime indicators that oscillatory roll vection was occurring.

The sessions were divided into two parts: first, a line-alone portion in which a truly oscillating line had to be nulled by proper steering-wheel movements; and second, a line-and-frame portion, in which only the frame oscillated at a $15^{\circ}$ amplitude, but in which the subject had to continuously null the illusory line oscillation. The line-alone or real-movement portion was divided into three 3.75min segments, each at a different peak line amplitude: the first was at $3.75^{\circ}$, the second at $7.5^{\circ}$, and the third at $15^{\circ}$. All three segments of the line-alone session were completed at the same frequency as the frame in the subsequent line-and-frame portion of the experiment. To maintain consistency, when a subject was tested in a circle condition, the line-alone portion as well as the line-andframe portion was completed with the circle illuminated. The lineand-frame portion lasted $25 \mathrm{~min}$. The static frame sessions entailed eight line-alone and eight line-and-frame settings, in which the frame was tilted $15^{\circ} \mathrm{cw}$ for half of the sessions and $15^{\circ} \mathrm{ccw}$ for the remaining ones.

\section{Dependent Measures}

For the analysis of the data taken during the sessions with oscillatory motion of the frame, the amplitude of the steering response was sampled at nine equally spaced points per oscillation cycle and averaged over the final 10 complete cycles of each condition. A best-fitting sinusoid was then calculated for these nine data points. The peak amplitude of this best-fitting sinusoid was the measure of illusory tilt. These response amplitudes were then converted into gains, the ratio of output to input amplitudes. A steering response equal in amplitude to that of the tilting frame would represent a perfect gain of 1.0. The ratio of an illusory line tilt of $3^{\circ}$ to a peak frame tilt of $15^{\circ}$ would correspond to a gain of 0.2 .

Phase shift averaged over the beginning and midpoint of each cycle was also calculated for these final 10 cycles. The phase shift was defined as the average number of degrees by which the steering response lagged behind the stimulus motion. For example, a phase shift of $-90^{\circ}$ would mean that the steering response was, on the average, a quarter cycle behind the movement of the frame.

In a similar fashion, gains and phase shifts were also calculated for the final 10 cycles in the control conditions, where only an oscillating line was present. The peak amplitude of these best-fitting sinusoids measured the ability of each subject to keep an actually oscillating line in an upright position.

\section{RESULTS}

The subjects who viewed the vertical line within the oscillating frame perceived the line itself to oscillate in opposition to the motion of the frame. For example, as the frame rotated clockwise, the enclosed line would appear to be rotating in a counterclockwise direction. For some individuals, the illusory line motion was quite dramatic, often $5^{\circ}$ to $10^{\circ}$ in peak amplitude during portions of the experimental session. However, as is often the case in rod-and-frame tasks, there were extreme individual differences. While all of the subjects did experience a kinetic RFE, a few perceived only a slight movement of the line, sometimes less than $1^{\circ}$ of peak tilt during particular frame cycles.

\section{Oscillatory Roll Vection}

Three different subjects reported periods of oscillatory roll vection during the frame oscillation. During complete oscillatory roll vection, the moving visual stimulus (the oscillating frame) appeared stationary, while the subjects perceived themselves to be rolling from side to side in a cartwheel fashion around their line of sight. The induced vection sensation, which was opposite to the direction of visual stimulus motion, lasted only about four to five cycles. Of potential significance is the fact that no reports of vection occurred when the circle circumscribed the oscillating frame. All three sessions with vection occurred at the highest frequency $(.213 \mathrm{~Hz})$ without the circle present.

\section{Kinetic Rod-and-Frame Effect}

Figure 1 shows the RFE measured in degrees, for conditions with and without a circular contour. A positive RFE value represents an error in line setting in the direction of frame tilt. For the entire sample of 9 subjects, the RFE of the kinetic frame tended to be larger than the RFE of the static frame condition. This was especially apparent at the lowest frame frequency $(.013 \mathrm{~Hz})$, where the kinetic RFE was $40 \%$ greater than the static RFE in the no-circle condition, yielding means of $3.96^{\circ}$ and $2.84^{\circ}$, respectively $[t(8)=2.29, p<.05]$. Corresponding values in the circle-present condition, where the mean static and slowest kinetic RFEs were $2.25^{\circ}$ and $3.03^{\circ}$, showed an increase of $35 \%$ in the kinetic condition $[t(8)$ $=3.18, p<.05]$.

A pattern similar to that of the nonvection subjects occurred in the data of the 3 vection-sensitive subjects, in that the latter also exhibited an increased effect at the

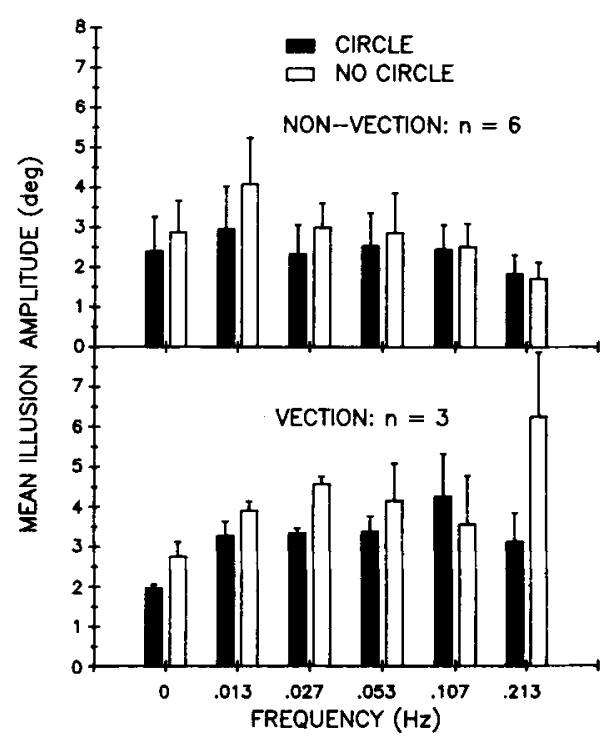

Figure 1. Mean illusion amplitude for static and sinusoidally oscillating frames: top, nonvection subjects; bottom, vection-sensitive subjects. Bar represents 1 SEM (standard error of the mean). 
lowest frequency over the static condition. The vectionsensitive subjects showed a higher RFE than the nonvection subjects at all but the static and lowest kinetic frequency, and they also exhibited an extraordinarily large RFE at the highest frequency, in the absence of the circle. This pattern produced a significant vection $\times$ circle $\times$ frequency interaction $[F(5,35)=5.64, p<.001]$.

\section{Orientation Inhibition}

Over all 9 subjects, the circle was effective in reducing the line-tilt illusion caused by the tilted frame $[F(1,7)$ $=17.95, p<.01]$. Averaged across all kinetic conditions, this reduction in tilt measured approximately $18 \%$. A similar inhibition of the RFE by the circle $(20 \%$ decrease) occurred for the static frame. The inhibitory effect of the circle was similar for both vection-sensitive and nonvection subjects, and, as Figure 2 suggests, it was limited to the three lowest frequencies. Further analyses of the orientation-inhibition effect will be presented below.

\section{Gain}

Vection-sensitive subjects. The 3 subjects reporting vection had very similar patterns of results. Figure 2 (right) shows their combined data. The gain data from the remaining 6 subjects are shown to the left in Figure 2 . A number of features distinguish these plots. One difference in the illusory-motion data is the sharp increase in the RFE gain at the highest frame frequency without the circle, for the subjects reporting vection. Note that this condition was the only circumstance in which self-motion was reported. In other words, the sessions with reports of vection yielded dramatic increases in the RFEapproximately a $77 \%$ increase in amplitude over the nearest frequency $[t(2)=6.58, p<.05]$. There was no overall effect of frame frequency on the response gains of the vection subjects, although a significant circle $\times$ frequency interaction was present $[F(4,8)=6.16$, $p<.01]$.

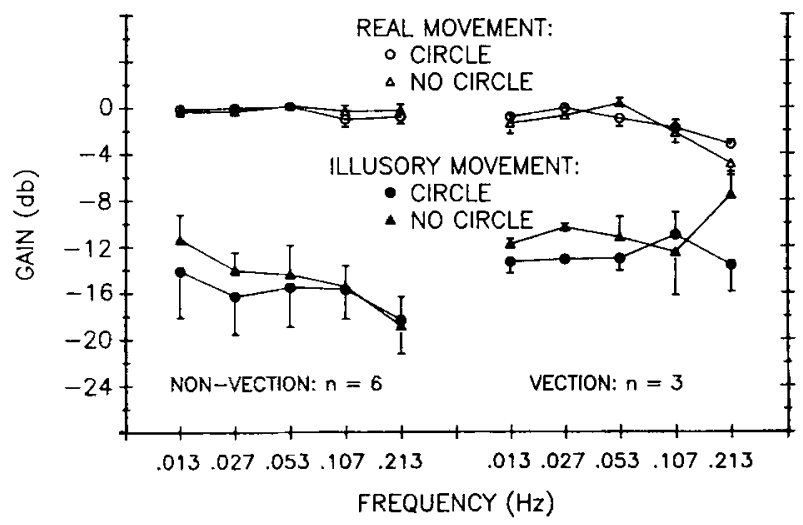

Figure 2. Gain as a function of frequency for real- (top) and illusory- (bottom) movement conditions, for nonvection subjects (left) and vection-sensitive subjects (right). Bar represents 1 SEM (standard error of the mean).

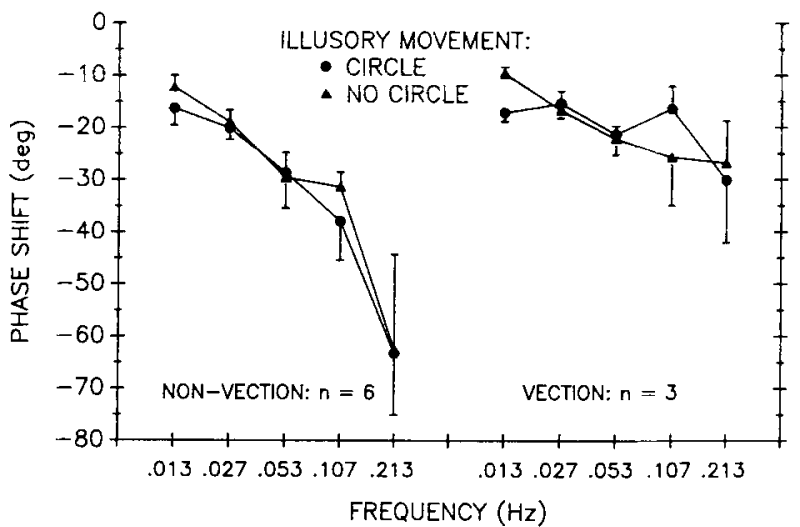

Figure 3. Phase lag as a function of frequency: illusory-movement conditions for nonvection subjects (left) and vection-sensitive subjects (right). Bar represents 1 SEM (standard error of the mean).

The top two functions to the right in Figure 2 represent the gain data from the control sessions with actual line motion. Only the real-motion condition with peak line amplitude of $3.75^{\circ}$ was analyzed fully, since this corresponded most closely to the obtained illusory amplitudes. Furthermore, visual analysis of all three real-motion amplitudes of 4 subjects at the highest and lowest frequencies showed an excellent linear relation between output (that is, the subjects' responses) and stimulus input amplitudes. Thus, the system was linear with respect to input amplitude and it may be concluded that the gain was identical, regardless of the stimulus amplitude chosen for analysis. With a peak line amplitude of $3.75^{\circ}$, the steering response during the control sessions was often nearly perfect, especially at the low line frequencies. It is potentially significant, however, that among the subjects reporting vection, the accuracy of the steering response decreased significantly as the line frequency increased $[F(4,8)=9.36, p<.01]$. A decrease in gain was most apparent above $.05 \mathrm{~Hz}$.

Nonvection subjects. The illusory-motion data for the nonvection subjects, shown to the left at the bottom of Figure 2, indicate a circle $\times$ frequency interaction, reflecting a greater inhibitory effect of the circle at the lower frame frequencies $[F(4,20)=3.08, p<.05]$, and, equivalently, a decrease in gain along with an increase in frequency-but mainly in the absence of the circle.

Under real line-movement conditions, represented at the top left of Figure 2, there was no significant change in the response gains across line frequencies. There was, furthermore, no difference in the steering response between circle and no-circle conditions during the control sessions for either the vection or the nonvection subjects.

\section{Phase Lag}

Nonvection subjects. To the left of Figures 3 and 4 are illustrated the phase-shift data for the 6 nonvection subjects with respect to illusory line motion and real line motion. The phase lag associated with the steering 


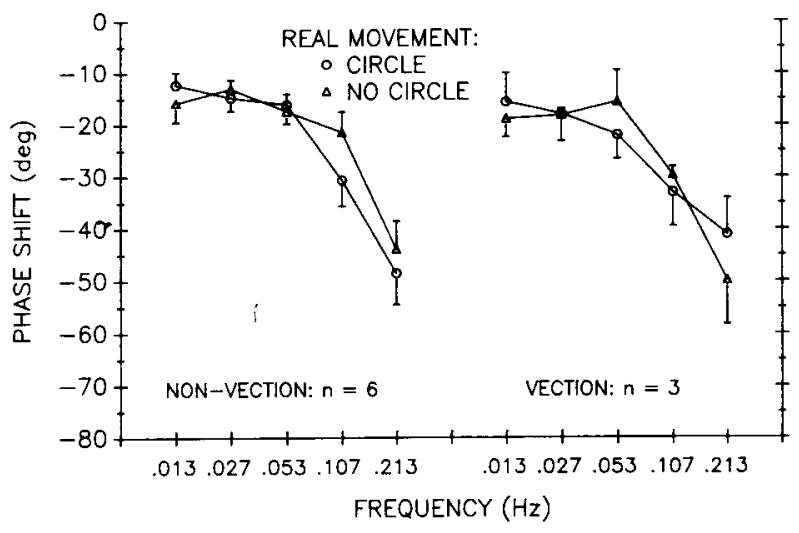

Figure 4. Phase lag as a function of frequency: real-movement conditions for nonvection subjects (left) and vection-sensitive subjects (right). Bar represents 1 SEM (standard error of the mean).

response for the nonvection subjects showed a steady increase as frequency increased, during both frame motion $[F(4,20)=7.14, p<.01]$ and actual line motion $[F(4,20)=21.87, p<.01]$. In other words, the steering response of the subjects lagged behind the movement of the visual stimulus. The phase shifts were the same with and without the circle. However, there was a greater phase lag during illusory line motion than during actual line motion $[F(1,5)=7.50, p<.05]$. The phase lag slowly increased from about $15^{\circ}$ at $.013 \mathrm{~Hz}$ to about $45^{\circ}$ at $.213 \mathrm{~Hz}$ during actual line motion. During illusory line motion, the phase lag increased from about $15^{\circ}$ to approximately $60^{\circ}$ at the highest frame frequency. The $60^{\circ}$ phase shift represents a steering response that trails the visual stimulus motion by one sixth of a cycle.

Vection-sensitive subjects. To the right of Figures 3 and 4 are shown the phase shift of the steering response during frame and actual line motion, for the subjects who reported vection. Under illusory-movement conditions, the shallow change in phase lag across frequencies, shown in Figure 3, was not statistically significant. In other words, the vection-sensitive subjects did not yield an increased phase lag, unlike the nonvection subjects, when the frame oscillated at high frequencies. The phase-shift data during the real-motion sessions, however, was quite similar for the vection-sensitive and the nonvection subjects, in that the former also yielded a significant increase in phase lag as frequency increased $[F(4,8)=15.75$, $p<.01]$. A marginally significant interaction between type of motion and frequency $[F(4,8)=3.82, p=.05]$

Table 1

Correlation Matrix Between Static Frame and Various Frequencies (Hz) of the Kinetic Conditions

\begin{tabular}{lllllll}
\hline & & \multicolumn{5}{c}{ Frequency } \\
\cline { 2 - 7 } & $n$ & .013 & .027 & .053 & .107 & .213 \\
\hline Nonvection subjects & 6 & .770 & .718 & .666 & .633 & .399 \\
All subjects & 9 & .755 & .579 & .629 & .545 & .175 \\
\hline
\end{tabular}

was also obtained, reflecting a significant increase in phase lag as frequency increased, but only under real-movement conditions.

\section{Static versus Kinetic RFE}

It has already been shown that the lowest-frequency kinetic RFE yields an RFE significantly higher than that of the static display. The question presently evaluated concerns the degree to which the static and kinetic conditions are correlated. A Pearson $\boldsymbol{R}$-correlation matrix, shown in Table 1, was prepared for the 6 nonvection subjects, eliminating the vection-sensitive subjects because of their anomalously enhanced performance at the highest frequency. None of the correlations reached statistical significance at $p=.05$, but the data suggest a clear trend toward a diminished degree of association as frame frequency (and peak velocity) increased. The data for all 9 subjects also show a great loss of association at the highest frequency. Although the correlation between the static pattern and the lowest-frequency kinetic condition this time achieved statistical significance at $p<.02$, none of the remaining correlation coefficients did likewise. This trend, because of the small sample size, is not conclusive, but it suggests a radical change in process as one responds first to a static frame and then to a high-speed kinetic version of the same pattern.

\section{DISCUSSION}

It has previously been suggested that the RFE represents a type of visual-vestibular interaction (Ebenholtz \& Glaser, 1982; Ebenholtz \& Utrie, 1983) similar to that witnessed during circular (Brandt, Dichgans, \& Koenig, 1973) and linear vection (Lestienne, Soechting, \& Berthoz, 1977), and most closely associated with roll vection (Held, Dichgans, \& Bauer, 1975). In fact, the RFE may be regarded as a static form of roll vection (Dichgans \& Brandt, 1978; Ebenholtz, 1977). These proposals are supported by the fact that oscillatory roll vection has actually been induced in some subjects during the oscillation of the frame. It has been concluded that while a stationary tilted framework may displace postural vertical (Ebenholtz \& Benzschawel, 1977), an oscillating frame may actually induce a continuously changing displacement, in both direction and degree, of apparent body orientation. Although a functional similarity thus has been identified between static and kinetic frame displays, the correlational analysis shown in Table 1 suggests that individual differences among observers are not maintained under the two conditions. The kinetic version seems to add an independent dimension, that of peripheral frame motion, so that performance on the two tasks becomes disassociated, especially at high frequencies or peak velocities. This result may help to explain the failure to adequately predict the incidence of motion sickness on flight simulators from performance on static rod-and-frame dis- 
plays (Frank \& Casali, 1986). It is also worth noting that although there is evidence for an enhanced dependence on visual stimulation for spatial orientation in microgravity environments (Oman, 1984; Young, Oman, Watt, Money, \& Lichtenberg, 1984), the capability to predict performance from earth to zero $\mathrm{G}$ may depend on the use of kinetic rather than static rod-and-frame tests.

It should be noted that only 3 of the 9 subjects experienced vection. Thus, perhaps because of the varying velocity and acceleration profile, a sinusoidally oscillating frame may be somewhat less effective in producing roll vection than other visual motion stimuli, such as continuously rotating constant-velocity random dot displays, are (Held et al., 1975). On the other hand, since vection was achieved only at the highest peak velocity $\left(20^{\circ} / \mathrm{sec}\right)$ employed in this study, higher velocities of frame oscillation may produce more frequent occurrences of oscillatory roll vection.

Why only certain individuals experienced sensations of self-motion during the frame movement remains of course a mystery. A clue may be provided by the high-frequency gain drop in the real line-movement condition (see Figure 2, right), for the individuals who reported vection. They apparently possess an increased sensitivity to motion in the central visual field, since they saturate earlytheir ability to process motion signals deteriorates at lower rotational velocities, relative to the ability of nonvection subjects. In contrast, the nonvection subjects showed a high and stable gain at all frequencies (see Figure 2, left). Thus sensitivity to optokinetic stimulation may be a predictor of vection proclivity and perhaps motionsickness sensitivity as well. It also may be significant that the phase-lag frequency functions differed for the vection and nonvection subjects, the former failing to show a significant phase-lag increase with increasing frequency under illusory-movement conditions. This suggests that vection-sensitive subjects have higher cutoff frequencies in peripheral vision: they tolerate higher velocities and have shorter time constants for the processing of motion stimuli in the retinal periphery than do nonvection subjects.

An interesting finding was the abrupt increase in the RFE during the sessions with reports of vection ${ }^{2}$ (see Figures 1 and 2). During conditions when one is most susceptible to visually induced self-motion, one may demonstrate a distinct increase in amplitude of an otherwise quite stable rod-and-frame effect. This suggests the operation of an orientation-constancy process (Ebenholtz, 1970), in which observers compensate for registered body tilt by rotating the line in the opposite direction. This process may combine with the effect of the frame tilt to produce an enhanced RFE. A relative increase in one's measured kinetic RFE, therefore, may provide a valuable indicator, which shows that conditions are especially conducive for vection.

If the RFE is, indeed, an instance of visual-vestibular interaction, then one would expect the kinetic RFE to act accordingly. For example, motion-sickness symptoms often accompany visual-vestibular conflicts (Lackner \& Graybiel, 1979), especially during moments of visually induced self-motion. Reports of dizziness and other discomforts were very frequent during the kinetic frame conditions, especially at the highest frame frequency, which was the condition in which vection sometimes occurred. Six of the 9 subjects reported dizziness during the highest frame frequency $(.213 \mathrm{~Hz})$. One potential subject was dropped from this study because of complaints of very severe motion-sickness symptoms (headache, nausea) while viewing the oscillating frame. No subject reported any discomfort during any of the control sessions in which the frame was absent.

As a group, the nonvection subjects with the circle absent exhibited a small but significant decrease in the RFE as the frame frequency increased. This small effect was not unexpected. For sinusoidal visual roll motion produced by oscillating random dot displays, the illusory tilt in verticality depends mainly on the oscillation angle of the visual stimulus (Mauritz, Dichgans, \& Hufschmidt, 1977). For random dot displays devoid of orientation information except that signaled via visual image motion, the oscillation angle or amplitude, as shown by Mauritz et al., is the product of the peak velocity and the oscillation period. The rod-and-frame apparatus, on the other hand, does have linear elements that in themselves signal tilt angle. Using a random dot display, Mauritz et al. found the amplitude of subjective vertical tilt to be directly proportional to the logarithm of the oscillation angle of roll motion. Certainly a roll motion stimulus of an oscillating frame may be expected to produce a similar relationship. Since, in the present study, the oscillation angle was maintained at a constant peak amplitude of $15^{\circ}$, an unchanging response gain should be expected on this basis. On the other hand, high frequencies tend to diminish response gains (Mauritz et al., 1977), whereas increasing peak velocities (correlated with frequency) tend to enhance the amplitude of the tilt illusion (Dichgans, Held, Young, \& Brandt, 1972; Held et al., 1975). As a result, any drop in gain that may be encouraged by a frequency increase would be nullified to some degree by the accompanying increase in the peak velocity of the visual stimulus. The absence of large gain loss with increasing frequency, therefore, is consistent with the conclusion that the oscillation angle of the roll motion stimulus is an important variable in the determination of the amplitude of illusory tilt in kinetic displays.

The nearly perfect response gains during the realmovement control sessions demonstrate the capability of the subjects in making steering movements that corresponded to the amplitude of actual line motion. The steering response during frame oscillation, therefore, may be considered a fairly precise measure of the amplitude of the illusory line movement. Nevertheless, a number of critical differences exist between the actual and apparent line movement. The actual line tilt during the control 
sessions stimulated receptors within about $8^{\circ}$ of the fovea, whereas illusory line movement during frame oscillation was a result of stimulation of receptors at about $30^{\circ}$ of eccentricity in the retinal periphery. ${ }^{3}$ Any illusory line movement caused by the oscillating frame would be localized in the area of the retina primarily reserved for the focal system (Leibowitz \& Post, 1982). Yet this apparent movement registered by the focal system was actually driven by peripheral stimulation. The need to develop an adequate physiological model for these events remains.

The inhibition effect of the surrounding circular contour on the rod-and-frame effect was extended to kinetic conditions. The circle around the oscillating frame was effective in reducing the rod-and-frame effect by about $21 \%$, averaged across the three lowest frame frequencies. This reduction, however, is considerably less than that found in a number of earlier studies (Ebenholtz, 1985b; Ebenholtz \& Utrie, 1983). All previous studies dealing with the inhibition of the RFE have tested the illusion only under static frame conditions. In this study, we also examined the inhibition effect of the circle with a stationary frame, but found only a $20 \%$ reduction of the RFE, compared to the average reduction of over $75 \%$ found by Ebenholtz and Utrie (1983). The fact that the RFE inhibition was relatively small under both static and kinetic conditions suggests that the dynamic nature of the oscillating frame was not the primary reason for the small inhibition under kinetic conditions.

One possible explanation for the relatively small inhibition effect is the extreme eccentricity of the frame and circle in the present experiment. In the present study, the diameter of the circle produced a retinal angle of $88^{\circ}$, which exceeded the retinal angle of $55^{\circ}$ used by Ebenholtz and Utrie (1983), or of $71^{\circ}$ in a more recent study (Ebenholtz, 1985b). It is generally the case that the RFE is enhanced as the frame moves toward the visual periphery (Ebenholtz, 1977; Ebenholtz \& Callan, 1980). For this reason, the apparatus was set at a distance that would produce an exceptionally large retinal angle. However, the circle may thereby have been placed too far into the periphery. In other words, the inhibition effect of the circle may depend nonmonotonically on its eccentricity. In the extreme periphery, the circle may be somewhat less effective than in less eccentric positions.

In addition to reducing the amplitude of the RFE, the circular contour also diminished the number of physical complaints reported by the subjects. For example, the number of sessions with reports of dizziness decreased by $38 \%$, from 13 to 8 , when the circle was present. Also, any occurrence of vection was totally eliminated by the presence of the circle. Consequently, a circular contour placed in the visual periphery may provide a means of suppressing motion-sickness symptoms that may arise from certain visual-vestibular conflicts (Ebenholtz \& Utrie, 1983). At the very least, a surrounding circle will suppress both static and low-velocity kinetic visual orien- tation information, which may otherwise produce changes in apparent self-orientation and perceived verticality.

\section{REFERENCES}

Brandt, Th., Dichgans, J., \& Koenig, E. (1973). Differential effects of central vs peripheral vision on egocentric and exocentric motion perception. Experimental Brain Research, 16, 476-491.

DichgaNs, J., \& BrANDT, Th. (1978). Visual-vestibular interactions: Effects on self-motion perception and postural control. In R. Held, H. Leibowitz, \& H.-L. Teuber (Eds.), Handbook of sensory physiology: Vol. VIII. Perception (pp. 755-807). New York: Springer-Verlag.

Dichgans, J., Held, R., Young, L. R., Brandt, Th. (1972). Moving visual scenes influence the apparent direction of gravity. Science, 138, 1217-1219.

Dichgans, J., Mauritz, K. H., Allum, J. H. J., \& Brandt, Th (1976). Postural sway in normals and atactic patients: Analysis of the stabilizing effects of vision. Agressologie, 17, 15-24.

Ebenholtz, S. M. (1970). Perception of the vertical with body tilt in the median plane. Joumal of Experimental Psychology, 83, 1-8.

EBENHOLTZ, S. M. (1977). Determinants of the rod and frame effect: The role of retinal size. Perception \& Psychophysics, 22, 531-538. EBENHoltz, S. M. (1985a). Absence of relational determination in the rod-and-frame effect. Perception \& Psychophysics, 37, 303-306.

Ebenholtz, S. M. (1985b). Depth separation fails to modulate the orientation-inhibition effect. Perception \& Psychophysics, 37, 533-535.

Ebenholtz, S. M., BenzschaWel, T. L. (1977). The rod and frame effect and induced head tilt as a function of observation distance. Perception \& Psychophysics, 22, 491-496.

Ebenholtz, S. M., \& Callan, J. W. (1980). Modulation of the rodand-frame effect: Retinal angle vs apparent size. Psychological Research, 42, 327-334.

EBenholtz, S. M., Glaser, G. W. (1982). Absence of depth processing in large-frame rod-and-frame effect. Perception \& Psychophysics, 32, 134-140.

EbENholtZ, S. M., \& Utrie, J. W., JR. (1982). Inhibition of the rodand-frame effect by circular contours. Perception \& Psychophysics, 32, 199-200.

Egenholtz, S. M., \& Utrie, J. W., JR. (1983). Peripheral circular contours inhibit the visual orientation control system. Aviation, Space, \& Environmental Medicine, 54, 343-346.

Frank, L. H., \& CaSali, J. G. (1986). Simulator sickness: A review of its costs, countermeasures, and prediction (Tech. Paper No. 861782). Warrendale, PA: Society of Automotive Engineers.

Held, R., Dichgans, J., \& Bauer, J. (1975). Characteristics of moving visual scenes influencing spatial orientation. Visual Research, 15, 357-365.

LaCKNER, J. R., GRaybiel, A. (1979). Some influences of vision on susceptibility to motion sickness. Aviation, Space, \& Environmental Medicine, 50, 1122-1125.

Leirowitz, R. W., Post, R. B. (1982). The two modes of processing concept and some implications. In J. J. Beck (Ed.), Organization and representation in perception (pp. 343-363). Hillsdale, NJ: Erlbaum.

Lestienne, F., Soechting, J., Berthoz, A. (1977). Postural readjustments induced by linear motion of visual scenes. Experimental Brain Research, 28, 363-384.

Mauritz, K. H., Dichgans, J., Hufschmid, A. (1977). The angle of visual roll motion determines displacement of subjective visual vertical. Perception \& Psychophysics, 22, 557-562.

Oman, C. (1984). Why do astronauts suffer space sickness? New Scientist, 8, 10-13.

Sigman, E., Goodenough, D. R., Flannagan, M. (1978). Subjective estimates of body tilt and the rod-and-frame test. Perceptual \& Motor Skills, 47, 1051-1056.

Sigman, E., Goodenough, D. R., Flannagan, M. (1979). Instructions, illusory self-tilt, and the rod-and-frame test. Quarterly Journal of Experimental Psychology, 31, 155-165. 
Streibel, M. J., Barnes, R. D., Julness, G. D., \& Ebenholtz, S. M. (1980). Determinants of the rod-and-frame effect: Role of organization and subjective contour. Perception \& Psychophysics, 27, 136-140.

WITKIN, H. A., \& Asch, S. E. (1948). Studies in space orientation: IV. Further experiments on perceptions of the upright with displaced visual fields. Joumal of Experimental Psychology, 38, 762-782.

Young, L. G., Oman, C. M., Watt, D. G. D., Money, K. E., \& LiCHTENBERG, B. K. (1984). Spatial orientation in weightlessness and readaptation to Earth's gravity. Science, 225, 205-208.

\section{NOTES}

1. Several subjects showed evidence of reflexive head-righting, in that head movements were made in phase with the frame. These data await further analysis.
2. It may be significant that the data, which were always taken on the last 10 cycles, happened not to be taken during the cycles in which vection actually was experienced, although the illusion amplitudes for vection and nonvection cycles appear not to differ.

3. The greater phase lag found for illusory conditions over that associated with real line movement may simply reflect the larger number of synapses entailed in orientation control via the periphery relative to the foveal and parafoveal retina.
(Manuscript received December 14, 1987; revision accepted for publication October 3, 1988.) 